\title{
Hyperdense middle cerebral artery sign
}

\author{
Gentle Sundar Shrestha ${ }^{1}$, MD, FCCP; Pankaj Jalan' ${ }^{2}$ MD \\ ${ }^{1}$ Department of Anaesthesiology, Institute of Medicine, Tribhuvan University Teaching Hospital, Kathmandu, Nepal \\ ${ }^{2}$ Department of Neurology, Norvic International Hospital, Kathmandu, Nepal
}

\section{Corresponding author}

Gentle Sundar Shrestha, MD, FCCP

Email: gentlesunder@hotmail.com

Received 29 Nov 2019

Accepted 20 Dec 2019

\section{Summary}

A 75 years old male patient, know case of hypertension and diabetes, presented to a rural hospital with the history of left sided weakness of 3 hours duration associated with facial deviation. Plain CT of head revealed increased density of right middle cerebral artery (MCA) - the hyperdense MCA sign (Panel A). There was associated hypoattenuation of right basal ganglia, insular ribbon sign and cortical swelling (Panel B). Patient was referred to a comprehensive stroke center due to lack of facility of thrombolysis or thrombectomy. Patient was intubated for drop in Glasgow Coma Scale to 7. Repeat CT head 8 hours after the initial scan showed persistence of hyperdense MCA sign (Panel C), infarction of right MCA territory and midline shift (Panel D). All the findings are indicated by arrows in the images. Patient underwent decompressive craniectomy followed by gradual recovery. Presence of hyperdense MCA sign is highly specific for obstruction of middle cerebral $\operatorname{artery}^{1,2}$.

\section{References}

1. Schuknecht B, Ratzka M, Hofmann E. The "dense artery sign" - major cerebral artery thromboembolism demonstrated by computed tomography. Neuroradiology 1990;32:98-103.

2. Jensen-Kondering $U$, Riedel $C$, Jansen $O$. Hyperdense artery sign on computed tomography in acute ischemic stroke. World J Radiol 2010;2:354-7. 


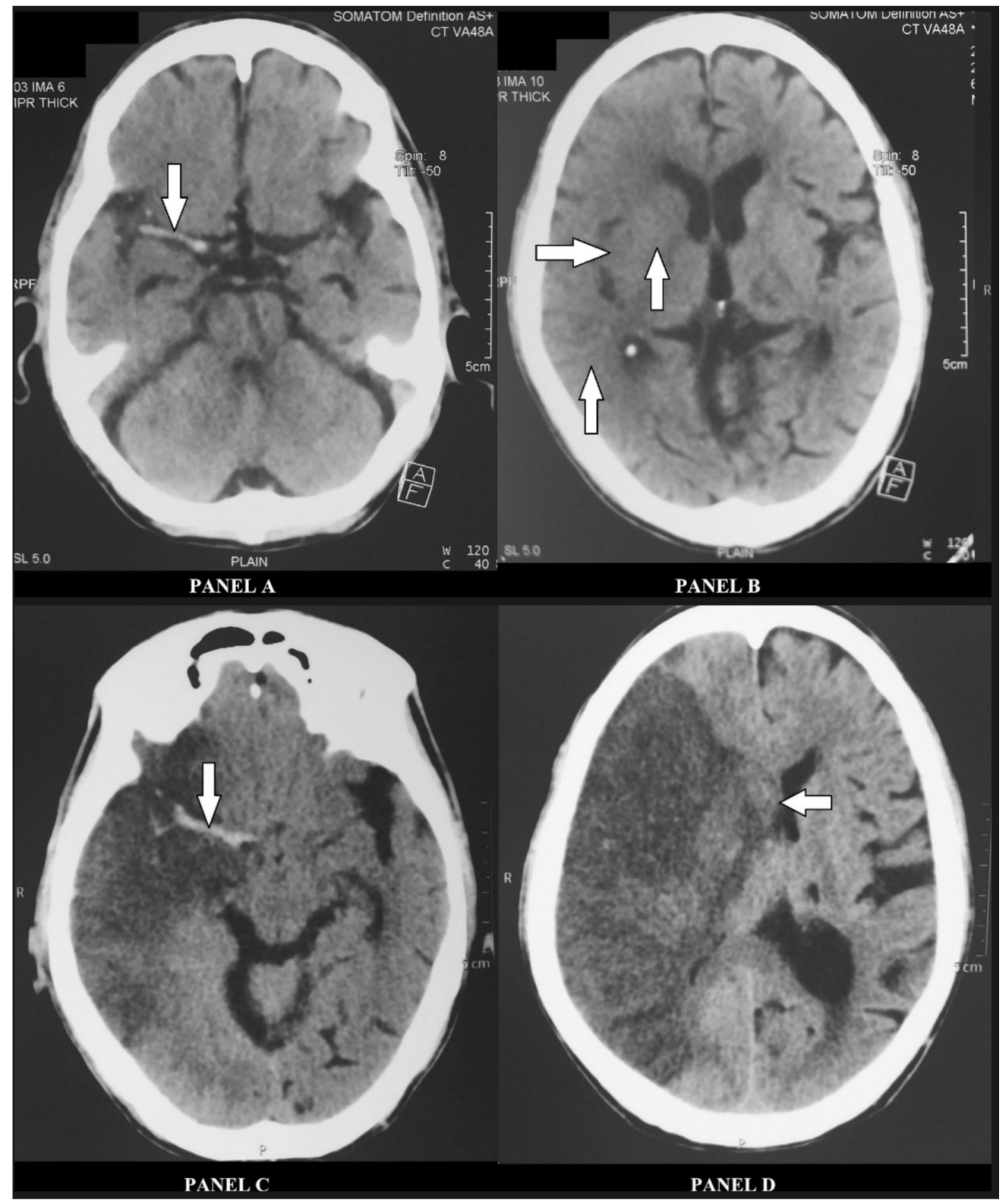

